\title{
Criminologie
}

\section{Situation risquée : le risque et les services correctionnels au Canada}

\section{Kelly Hannah-Moffat et Margaret Shaw}

Volume 34, numéro 1, printemps 2001

La notion de risque dans la gestion pénale

URI : https://id.erudit.org/iderudit/004755ar

DOI : https://doi.org/10.7202/004755ar

Aller au sommaire du numéro

\section{Éditeur(s)}

Les Presses de l'Université de Montréal

ISSN

0316-0041 (imprimé)

1492-1367 (numérique)

Découvrir la revue

Citer cet article

Hannah-Moffat, K. \& Shaw, M. (2001). Situation risquée : le risque et les services correctionnels au Canada. Criminologie, 34(1), 47-72.

https://doi.org/10.7202/004755ar
Résumé de l'article

Les auteurs avancent que la tendance à généraliser la gestion du risque comme une caractéristique répandue dans les sociétés modernes néglige la spécificité des constructions, des histoires et des applications particulières du terme risque dans un contexte social donné. L'article s'interroge sur le concept de risque dans le cadre de la recherche et de la politique du gouvernement fédéral canadien en matière correctionnelle. Plus particulièrement, il traite de la façon dont la construction du risque dans la recherche correctionnelle et dans les politiques visant l'évaluation et la gestion de tels risques fait référence à une norme d'homme blanc, de classe moyenne, ou, en d'autres mots, comment le risque est défini selon le genre et la race. Il aborde également la manière dont l'hybridation du risque et de la réhabilitation (évidente dans les concepts d'évaluation risque/besoin et de gestion risque/besoin) contribue à la réaffirmation de la réhabilitation, faisant ainsi apparaître un modèle mixte de gestion. 


\title{
Situation risquée : le risque et les services correctionnels au Canada ${ }^{1}$
}

\author{
Kelly Hannah-Moffat \\ Professeur \\ Département de sociologie \\ Université de Toronto \\ khmoffat@credit.utoronto.ca \\ Margaret Shaw \\ Professeur \\ Département de sociologie et d'anthropologie \\ Université Concordia \\ mshaw@vax2.concordia.ca
}

\begin{abstract}
Comment comprendre notre société si nous transformons le concept du risque en un problème universel qu' on ne pourra ni éviter, ni éluder?
\end{abstract}

LUHMANN, I993: IX.

RÉSUMÉ - Les auteurs avancent que la tendance à généraliser la gestion du risque comme une caractéristique répandue dans les sociétés modernes néglige la spécificité des constructions, des histoires et des applications particulières du terme risque dans un contexte social donné. L'article s'interroge sur le concept de risque dans le cadre de la recherche et de la politique du gouvernement fédéral canadien en matière correctionnelle. Plus particulièrement, il traite de la façon dont la construction du risque dans la recherche correctionnelle et dans les politiques visant l'évaluation et la gestion de tels risques fait référence à une norme d'homme blanc, de classe moyenne, ou, en d'autres mots, comment le risque est défini selon le genre et la race. Il aborde égale-

1. Nous aimerions remercier Condition féminine Canada pour le financement d'un projet de recherche de deux ans (Gender, Diversity, Risk Assessment And Classification For Federally Sentenced Women) et les participants au Workshop on Risk de mai 1999. Les données recueillies pour ce projet ont éclairé notre réflexion sur les pratiques actuelles. Cet article est également disponible en anglais.

Criminologie, vol. 34, $n^{\circ} 1$ (2001) 
ment la manière dont l'hybridation du risque et de la réhabilitation (évidente dans les concepts d'évaluation risque/besoin et de gestion risque/besoin) contribue à la réaffirmation de la réhabilitation, faisant ainsi apparaître un modèle mixte de gestion.

ABSTRACT - The authors argue that the tendency to generalize risk management as a common feature of modern societies, overlooks the specificity of particular constructions, histories and applications of the term risk in a given social context. This article is concerned with the concept of risk as it relates to current Canadian federal correctional research and policy. In particular, how the construction of risk in correctional research and in policies targeting the assessment and management of such risks evokes a white, middle class, male norm or in other words, how risk is gendered and racialised. And further, how the hybridisation of risk and rehabilitation (evident in the concepts of risk/need assessment and risk/need management) contributes to the reaffirmation of rehabilitation.

Le risque, sa gestion et son évaluation constituent un problème primordial autant pour les théoriciens, les praticiens, les responsables des politiques, que pour les entreprises commerciales et les simples citoyens. Les gouvernements se voient de plus en plus confrontés à l'obligation d'identifier le risque et d'en minimiser la progression (O'Malley, 1999). De nos jours, le risque revêt la forme d'un mantra déterminant dans nombre de travaux portant sur le système pénal. Les rationalités et les technologies du néo-libéralisme, qui prétendent mesurer et définir avec exactitude le risque, se retrouvent très présentes dans le système pénal canadien. Largement théorique, la littérature sur le thème du risque affirme qu'on voit actuellement apparaître une société fondée sur le risque, et que la gestion des individus et des populations repose de plus en plus sur les techniques actuarielles de gestion du risque (Simon, 1988, 1987 ; Castel, 1991 ; Defert, 1991 ; Ewald, 1991 ; O’Malley, 1992, 1998, 1999 ; Feeley et Simon, 1992, 1994 ; Simon et Feeley, 1995 ; Parton, 1996 ; Pratt, 1997 ; Ericson et Haggerty, 1997 ; Lupton, 1999 ; Dean, 1999 ; Culpitt, 1999 ; Hannah-Moffat, 1999).

Selon Culpitt (1999: 3), le risque n'est plus simplement associé au destin personnel ; il revêt désormais un visage public, envahissant, irrésistible et inévitable. L'auteur suggère de plus que les discours sur le risque ne représentent pas un point fixe d'analyse que l'on peut ramener à estimation, prédiction et conséquence. Il considère plutôt le risque comme une partie intégrante de la façon dont nous définissons «le personnel et le social» (Culpitt, 1999 : 10). En conséquence, Culpitt affirme que les analyses du risque dans son rapport avec les politiques sociales doivent prendre en considération des discours politiques plus amples au sujet de l'élaboration et du maintien d'idéologies sur le besoin et le bien-être 
(Culpitt, 1999 : 10). Bien des spécialistes déclarent qu'il existe un lien complexe entre le concept du risque et les politiques sociales (Dean, 1999), et qu'il mérite une analyse plus approfondie. Alors que bien des analyses du risque se sont penchées sur l'innovation d'une gestion fondée sur le risque, peu de ces analyses ont considéré comment le risque interagit avec les autres formes de gestion, les domaines où le risque se développe fort aisément, les hybrides qui se forment et la façon dont on les gère, ainsi que les conditions qui restreignent l'exploitation et la croissance des technologies fondées sur le risque (O'Malley, 1999 : 14). On peut alors se demander comment une gestion fondée sur le risque peut être associée à d'autres modalités de gestion, comme celle de l'État-providence, dans la prise en charge des populations correctionnelles.

Ewald (1991 : 191) affirme que «rien n'est un risque en soi ; le risque ne fait pas partie de la réalité. Cependant, tout peut constituer un risque ; tout dépend de quelle façon on analyse le danger, on considère la situation ». Notre intérêt réside dans ce que l'on considère comme risque, comment on le définit et l'identifie. Dans un contexte pénal, le terme risque possède de multiples significations. Le désir ou le besoin d'une évaluation du risque dans la pratique correctionnelle actuelle est urgent. Toutefois, il n'y a pas vraiment consensus sur la signification ou le type de risque à prédire et à gérer. Le discours correctionnel sur le risque à l'heure actuelle ramène en surface un vieux débat sur l'identification et la gestion des individus dangereux ${ }^{2}$ et la détermination de « ce qui fonctionne » en termes d'interventions correctionnelles. On se préoccupe particulièrement de la possibilité qu'un individu vienne perturber l'ordre institutionnel et, en fin de compte, du risque de récidive. Le vocabulaire du risque qu'on évoque présentement dans le discours correctionnel prétend être objectif, efficace et fondé sur des techniques actuarielles de gestion de population. La gestion du risque se révèle dans le déploiement de techniques actuarielles objectives conçues pour identifier et minimiser les risques. Les techniques actuelles de correction attirent l'attention sur la responsabilisation ${ }^{3}$ des délinquants en insistant pour qu'ils « assument la responsabilité de leurs actes ».

2. Pour des analyses supplémentaires sur la dangerosité et sa gestion jusqu'à présent voir Pratt, 1993, 1997 et dans ce numéro.

3. Ce terme s'applique à la charge de responsabilité de gestion du risque que l'État a remis entre les mains des individus, communautés et familles, qui sont conseillées, formées et tenues responsables de la gestion prudente et raisonnable des risques (O'Malley, 1999 ; voir aussi O'Malley, 1998). 
Nous sommes d'avis que la tendance à généraliser la gestion du risque comme une caractéristique répandue dans les sociétés modernes néglige la spécificité des constructions, des histoires et des applications particulières du terme risque dans un contexte social donné. Le présent article s'interroge sur le concept du risque dans le cadre de la recherche et de la politique du gouvernement fédéral canadien en matière correctionnelle. Plus particulièrement, comment la construction du risque dans la recherche correctionnelle et dans les politiques visant l'évaluation et la gestion de tels risques fait référence à une norme d'homme blanc, de classe moyenne, ou, en d'autres mots, comment le risque est défini selon le genre et la race. Et également, comment l'hybridation du risque et de la réhabilitation (évidente dans les concepts d'évaluation risque/besoin et de gestion risque/besoin) contribue à la réaffirmation de la réhabilitation, faisant ainsi apparaître un modèle mixte de gestion.

\section{Risque et classification correctionnelle}

À partir de la fin des années 1970, tant en Amérique du Nord qu'ailleurs, les politiques de classification correctionnelle prennent un nouveau tournant. Dans plusieurs prisons, l'objectif principal du système de classification est passé de la sécurité au risque et à sa gestion (i.e. évasion, risque pour la population, pour les autres prisonniers, pour le personnel, pour les institutions, pour les délinquants eux-mêmes) principalement par le moyen de stratégies de neutralisation. Les méthodes pour évaluer le risque et prédire les comportements à risque se sont aussi transformées ${ }^{4}$. Les listes de contrôle et les évaluations cliniques autrefois en usage sont maintenant considérées comme des méthodes de classification subjectives et discrétionnaires qui doivent être remplacées par des outils objectifs et des mesures actuarielles qui créent des réponses standardisées et des profils de risque provenant d'études sur de larges échantillons de population (Gottfredson et Tonry, 1987 ; Dallao, 1997). On considère que ces outils vont éliminer les prises de décision arbitraires, les partis pris et les préjugés, pour révéler une classification plus efficace et impartiale, et des institutions empreintes de rationalité et de justice. On affirme donc que ces outils sont meilleurs pour prédire le risque et la récidive que les mesures antérieures. La prédiction actuarielle du risque est maintenant

4. Suite à l'établissement des lignes directrices sur la détermination de la peine et la libération conditionnelle. 
en vigueur dans nombre de systèmes carcéraux ou de libération conditionnelle en Occident, outre le Canada et les États-Unis, soit en Angleterre et au pays de Galles (Clark, Fisher, McDougall, 1993 ; Ditchfield, 1997 ; Mair, 1999), de même qu'en Australie (Brown, 1996 ; Daley et Lane, 1999 ; Dawson, 1999) ; d'autres types de services sociaux subissent le même genre de modification (cf. Castel, 1991).

Une gestion fondée sur le risque présente diverses caractéristiques qui la rendent attrayante et compatible avec les orientations correctionnelles bureaucratiques de sécurité. Comme le constate O'Malley (1999 : 1) une gestion fondée sur le risque :

- est orientée vers l'avenir ou prédictive, et adaptée à la minimisation des torts;

- se préoccupe de cas ou de situations individuelles en tant qu'éléments de catégories de risque ;

- se préoccupe surtout d'analyser les statistiques du risque plutôt que de se pencher sur les problèmes individuels ;

- semble plus technique, efficace, objective et non moralisatrice ;

- ne cherche pas à identifier les causes mais plutôt à repérer les facteurs spécifiques du risque.

Jusqu'à un certain point, ces caractéristiques sont présentes dans le discours, les politiques ou la pratique en matière correctionnelle au Canada.

Les mesures actuarielles font partie d'une tendance croissante visant la gestion des cas, l'efficacité et l'obligation de rendre compte dans les systèmes correctionnels, s'éloignant de l'intérêt pour les cas individuels (Feeley et Simon, 1994 ; Garland, 1996). Ces mesures servent à améliorer la prise de décision individuelle, le jugement ou les décisions cliniques. On prétend qu'elles réduisent les prises de décision improvisées et entrainent une uniformité et une équité plus grandes dans les décisions. Elles sont plus efficaces pour déterminer l'environnement institutionnel le mieux adapté à l'individu, et pour mettre en œuvre services et programmes. Ces mesures répondent également à l'obligation de rendre compte et aux besoins de gestion pour le personnel pénitentiaire. Au Canada, l'identification et la gestion du risque sont au centre des préoccupations des chercheurs en matière correctionnelle, des administrateurs du système pénal, de la Commission des libérations conditionnelles et des gens qui travaillent en milieu correctionnel communautaire. Ce constat est particulièrement évident dans le système pénitentiaire fédéral et dans la recherche correctionnelle gouvernementale qui s'y rapporte. Les questions relatives à l'évaluation et à la gestion du risque se révèlent clairement dans les 
débats et les politiques reliées à la classification relative à la sécurité, aux évaluations d'admission, à l'établissement des programmes et à la planification des remises en liberté (sécurité publique). Chacun de ces points implique une construction sociale du risque que le délinquant représenterait pour le public, pour lui-même ou pour l'institution. Reliées à ces constructions, il existe des interventions destinées en théorie à minimiser le risque que présente le délinquant et à le gérer.

Au Canada, Price suggère que « l'enthousiasme des gens qui réalisent des évaluations du risque frôle le culte. Les personnes qui traitent des questions d'éthique, de loi ou des implications du système brillent par leur absence » (Price, 1997 : 2). D'autres auteurs ont avancé que le concept $\mathrm{du}$ risque est passé du risque raisonnable à celui de l'absence de risque, affirmant que les responsables de l'évaluation des cas passent plus de temps devant leurs ordinateurs qu'en interaction avec les détenus ou les individus en liberté conditionnelle (CCJA, 1998).

\section{Fusionner risque et réhabilitation : un modèle mixte de gestion}

Au Canada, l'accent mis sur le risque dans la recherche correctionnelle des dernières années s'intègre nettement à une stratégie plus vaste de réaffirmation de la réhabilitation en démontrant «ce qui fonctionne » en termes d'intervention. Dans les années 1990, on remettait l'accent sur le traitement et sur la réhabilitation. On intégra le risque aux politiques de réhabilitation. L'analyse des facteurs de risque a été reliée à l'identification des facteurs criminogènes ${ }^{5}$ qui jouent un rôle dans la prévention plutôt que dans la simple prédiction du délit. On utilise désormais les outils actuariels pour classifier les détenus non pas seulement par rapport aux risques qu'ils présentent pour la sécurité, mais également en termes de leurs besoins criminogènes. Les outils d'évaluation du risque tiennent un rôle primordial pour associer « les niveaux de traitement au niveau de risque du délinquant » (Andrews, Bonta et Hoge, 1990). Au Canada, une grande partie du travail accompli relève des psychologues en milieu correctionnel (Andrews, Bonta et Hoge, 1990 ; Andrews, Zinger, Hoge, Bonta, Gendreau et Cullen, 1990). Ces derniers sont convaincus qu'on ne peut réduire la récidive que par

5. Cette construction du facteur criminogène est semblable à ce que O'Malley (1999) nomme facteur protecteur. 
certains types bien spécifiques de traitements (qui relèvent de la psychologie cognitive), et seulement lorsque appliqués à certains groupes bien définis de délinquants. De la classification du risque et des besoins découle donc une classification au niveau de la sécurité, de même que l'attribution du niveau de traitement ou de supervision. Plus récemment, on a modifié la terminologie ; on ne parle plus de risque criminogène mais de facteurs statiques immuables (comme l'âge ou les délits antérieurs), ni de besoin criminogène mais de facteurs dynamiques, que les programmes de traitement sont en mesure de modifier.

Nonobstant le scepticisme qui règne en criminologie et dans les autres sciences sociales au sujet de notre capacité à établir des prédictions précises et fiables quant à la dangerosité et à la récidive, les chercheurs canadiens soutiennent qu'il existe un lien étroit entre le type et le nombre de besoins que présentent les délinquants et la probabilité de récidive ; ils affirment également qu'une évaluation combinée du risque et des besoins va améliorer notre capacité à prédire quels délinquants sont plus susceptibles de récidiver (Motiuk, 1993). L'identification de ces risques et besoins découle de recherches qui évaluent la probabilité d'une future implication criminelle. Les tentatives des chercheurs pour identifier et quantifier risque et besoins ont abouti à une nouvelle conceptualisation des besoins et de certaines barrières sociales structurelles en tant que facteurs de risque. Cette récente tendance reflète une hybridation du risque et de la réhabilitation. Il s'agit d'un "modèle mixte de gestion $»^{6}$ dans lequel on réaffirme et on redéploie les stratégies de réhabilitation pour minimiser et réduire le risque. Cette tendance remet en place les programmes de réhabilitation au sein des pénitenciers.

Dans le cadre actuel du discours correctionnel et des pratiques d'évaluation du risque, ce modèle mixte de gestion a entraîné un dérapage substantiel entre les concepts de risque et de besoin. Un besoin non satisfait entraînerait, semble-t-il, un facteur de risque potentiel. Dans certains cas, on ne peut distinguer l'un de l'autre. Selon Hannah-Moffat (2000 : 36) :

la fusion du risque et du besoin crée un paradoxe intéressant, combinant deux éléments plutôt différents : les soucis traditionnels de sécurité, généralement associés au danger et à la prévention de tort à autrui, et un accent plus récent sur le besoin, qui, par contraste, implique que le prisonnier manque de quelque chose et qu'il a droit à des ressources.

6. Voir O'Malley (1999) pour une discussion plus en profondeur des " modèles mixtes de gestion" découlant du jumelage du risque et de la peine, du risque et de la justice réparatrice, et du risque et de la réhabilitation. 
Par conséquent, les besoins criminogènes sont explicitement définis comme des problèmes qui influencent les probabilités de récidive, plutôt qu'une déclaration de droits (Hannah-Moffat, 1999). Toutefois, la littérature canadienne ne perçoit pas tous les besoins comme criminogènes. On ne retrouve pas cet accent sur le besoin criminogène et la réhabilitation dans la plupart des autres systèmes correctionnels occidentaux (Garland, 1996).

Dans la littérature canadienne récente sur la politique de classification (particulièrement pour les femmes délinquantes), on retrouve souvent le terme hybride risque / besoin, et certaines caractéristiques du délinquant sont à la fois identifiées comme risques et comme besoins. On inclut parmi les caractéristiques définies comme besoins criminogènes la dépendance, une faible estime de soi, de piètres résultats au niveau scolaire ou professionnel, la mort des parents tôt dans la vie du délinquant, le placement en foyer d'accueil, les changements incessants de foyer d'accueil, le placement en résidence, la vie dans la rue, la prostitution, les tentatives de suicide, l'automutilation, l'abus de stupéfiants, et les responsabilités parentales (FSWP, 1994 : 5). Plus récemment, on a inscrit au nombre des facteurs de risque pour les femmes délinquantes la victimisation à répétition et des comportements d'automutilation à l'âge adulte (Bonta et al., 1995 ; Blanchette, 1997a, 1997b). L'hybridation des risques et des besoins a entrainé l'identification d'une « multitude de facteurs de risque non reliés et qui ne permettent pas de poser des assises pour des interventions systémiques de réhabilitation » (O'Malley, 1999 : 18). L'effondrement des catégories risque et besoin et la formulation des besoins en tant que risques amènent à redéfinir la gestion des femmes et des populations marginalisées. Cette nouvelle formulation implique également la responsabilité individuelle de la gestion du risque plutôt que le droit aux programmes et services, tout en justifiant et en réaffirmant simultanément l'importance des programmes de réhabilitation en tant que stratégies visant à minimiser le risque de récidive.

\section{Nature subjective du risque : le système de classification fédéral actuel}

L'évaluation initiale des délinquants, instaurée en 1994 dans huit pénitenciers canadiens, y compris la Prison des femmes (Motiuk, 1997), est présentement en vigueur dans tous les établissements fédéraux au moment de l'admission (incluant les institutions pour femmes et le 
Pavillon de ressourcement pour autochtones). Cette évaluation a été mise au point et validée sur la population mâle (à prédominance) blanche des pénitenciers fédéraux. On décrit le processus d'évaluation à l'admission comme une « analyse systématique et opportune d'information importante, prédictive du risque et des besoins ». Outre l'estimation du niveau de sécurité et du placement en pénitencier, ce processus détermine le risque et les besoins de traitement avec « une liste par priorités des besoins reliés à la réduction du risque de commettre à nouveau un délit » (CSC, 1996).

On décrit cette évaluation comme une approche multidisciplinaire, multi-méthodes et multi-sources à la classification ${ }^{7}$. On utilise la portion risque de l'évaluation pour déterminer les classifications de sécurité ; les évaluations de besoins (scolarité, emploi, abus de substances) devraient être indépendantes de la classification de sécurité, quoique essentielles pour établir le plan correctionnel approprié. Le plan correctionnel devrait définir une série de programmes qui, s'ils sont appliqués, pourraient aider à réduire la probabilité de récidive. Idéalement, à la fin du processus d'évaluation, les autorités pénitentiaires devraient avoir une compréhension approfondie du risque que pose le délinquant pour la communauté, des facteurs qui l'ont conduit à la criminalité, des domaines de besoins à traiter pour réduire le risque d'une nouvelle infraction, et de l'institution ${ }^{8}$ qui convient le mieux à ce délinquant. L'élément évaluation des besoins de l'évaluation initiale concerne sept domaines : emploi, mariage/famille, pairs/ interaction sociale, abus de substances, fonctionnement dans la communauté, orientation personnelle/émotionnelle, et attitudes. Les réponses à quelque 250 questions qui permettent d'opérationnaliser davantage ces variables fournissent de l'information sur chacun de ces domaines. À ce jour, personne n'a encore modifié ces questions pour tenir compte du genre du délinquant, des éléments ethno-culturels ou des contextes socio-économiques.

Ainsi, le but principal de cet outil consiste à identifier les domaines de la vie du délinquant (facteurs de risque/besoin criminogènes) qui requièrent une intervention pour en réduire le risque, et pour concevoir un plan correctionnel qui vise ces domaines à risque élevé. Par conséquent, le délinquant sera dirigé selon son appartenance à certaines caté-

7. Information tirée du Offender Intake Assessment and Correctional Plan - User's Manual.

8. Les options de placement institutionnel pour les femmes sont restreintes aux prisons régionales à multiples niveaux. La gamme des options d'hébergement pour hommes est différente de celle des femmes. 
gories de risque précises, imputées à tort à l'individu et établies comme s'il existait une relation causale entre elles et la conduite future probable. La légitimation des programmes correctionnels réside dans leur capacité à minimiser les risques futurs en réformant l'individu. Les décisions administratives au sujet de la gestion de la sentence du délinquant dépendent grandement de la volonté et de la capacité du délinquant à «prendre conscience » de ses propres facteurs criminogènes, et à aborder ces facteurs positivement grâce aux programmes, de façon à ce que le risque que ce délinquant pose puisse être géré dans une institution à sécurité réduite et, finalement, dans la communauté. Le fait de ne pas se soumettre à la stratégie de réduction/gestion du risque peut entraîner, par exemple, le transfert dans une institution à sécurité maximale, ou le refus de la libération conditionnelle.

Ces échelles exigent l'intervention d'experts pour poser une foule de jugements moraux concernant le passé et l'avenir probable du délinquant. Les gens qui élaborent les échelles d'évaluation du risque ne sont pas nécessairement les praticiens qui utilisent ces outils ${ }^{9}$. L'utilisation et l'interprétation des outils sont essentiels pour comprendre les complexités d'une gestion fondée sur le risque. Des outils comme l'évaluation initiale exigent des experts pour faire une évaluation subjective des habitudes de loisirs (ou l'absence d'habitudes de loisirs socialement acceptables), de la qualité des relations, des attitudes du délinquant, de même que de sa stabilité professionnelle et de son niveau de scolarité ${ }^{10}$. Ces critères d'évaluation représentent les normes morales et sociales de la classe moyenne de race blanche. L'incapacité de se conformer à ces normes implique une déviation inacceptable de la norme. Par exemple, les variables servant à évaluer le fonctionnement dans la communauté et les interactions sociales comprennent respectivement « la participation à des activités organisées (équipes sportives, associations et groupes paroissiaux) » et «sans lien avec des groupes communautaires (organismes de charité, Grands frères, groupes d'athlétisme) ». De telles catégorisations requièrent des évaluations normatives des habitudes de loisirs, auxquelles bien des citoyens honnêtes ne répondent pas.

9. Une certaine disparité entre l'intention de la personne qui met au point un outil et son usage dans la pratique est chose courante. Toutefois, malgré cet écart, nous maintenons que ces outils, mêmes utilisés correctement, imposent certaines normes.

10. Les exemples cités sont tirés du Offender Intake Assessment And Correctional Plan User's Manual produit par le Service correctionnel du Canada. 
Les critères servant à indiquer «l'orientation personnelle et émotionnelle » d'un délinquant requiert également une évaluation subjective du comportement qui pourra refléter des constructions basées sur le statut social, la culture d'origine ou le genre de l'individu. Par exemple, des indicateurs tels que "l'ethnicité est problématique » ou « la religion est problématique » peuvent être pour beaucoup d'autochtones le reflet d'expériences racistes ou d'expériences religieuses négatives. De la même façon, des indicateurs tels que "identité sexuelle considérée comme problématique par le délinquant», "préférences sexuelles inappropriées » (voulant dire que les préférences sexuelles ne sont pas conformes aux normes sociales), «comportements sexuels problématiques » (voulant dire que les attitudes sexuelles du délinquant ne sont pas conformes aux normes sociales) peuvent insinuer que l'hétérosexualité est la norme et que les préférences sexuelles non traditionnelles constituent un comportement déviant.

On retrouve des jugements semblables dans les évaluations des relations familiales ou maritales, où la norme implique une famille nucléaire stable et hétérosexuelle, et où ce sont les mères qui sont principalement chargées des soins. On trouve peu d'indications sur la façon d'évaluer les relations familiales en milieu ethnique qui reposent sur la famille élargie. Voici quelques critères utilisés dans l'évaluation initiale pour évaluer les relations maritales ou familiales : enfance dépourvue de liens familiaux, mère ou figure maternelle absente durant l'enfance, père ou figure paternelle absente durant l'enfance, présentement célibataire, responsabilités parentales, capacité à contrôler son comportement de façon « appropriée », compréhension du développement de l'enfant, programmes sur le mariage / la famille suivis en entier. Ces critères servent à évaluer tous les délinquants, sans tenir compte de leur genre, de leur race ou de leur pertinence avec les convictions du délinquant.

Les évaluations quasi actuarielles du risque comprennent non seulement les catégories antérieurement construites comme besoins, mais aussi une série de variables sociales structurelles sur lesquelles le délinquant a peu, sinon pas du tout, d'influence. Alors que l'inclusion ou l'exclusion de variables dans une échelle donnée est souvent le résultat d'un processus complexe, l'inclusion de certaines variables, la tendance à individualiser, et l'incapacité à tenir compte du contexte et à qualifier les évaluations du risque peuvent reproduire des inégalités sociales plus grandes, et désavantager injustement certains groupes sociaux. L'exposition à certaines conditions sociales, volontairement ou non, se retrouve 
dans les déterminations institutionnelles du niveau de risque d'un délinquant et dans la capacité future à gérer et à minimiser ce risque. Les techniques actuarielles évaluent le risque au niveau individuel; on ne peut donc pas considérer comme des indicateurs prévisionnels valables des variables structurelles sociales plus larges (pauvreté, racisme, pouvoir discrétionnaire de la police) qui affectent indirectement le risque de récidive ou les comportements en institution (structure institutionnelle, accès aux programmes, comportement du personnel, comportement discrétionnaire des professionnels) et sur lesquels les programmes se penchent rarement. Par exemple, bien des évaluations correctionnelles $\mathrm{du}$ risque incluent des variables telles que "vit dans un milieu criminogène » ou « logement instable » (c'est-à-dire déménage souvent, soit plus d'une fois par année). Ces situations sont probablement reliées à la pauvreté et à l'incapacité de trouver un logement abordable en milieu urbain, plutôt que le signe d'un refus d'adopter un mode de vie moins criminalisé. Les questions que renferment les outils d'évaluation tels l'évaluation initiale et auxquelles on doit répondre par oui ou non, comme : «n'a pas de compte en banque », «n'a pas de garants », «n'a pas de crédit» comme indication du « fonctionnement au sein de la communauté », ne tiennent pas compte de la pauvreté et de la situation sociale. Alors qu'il est difficile sinon impossible pour les outils de profil du risque d'intégrer les variables sociales structurelles, il est essentiel de reconnaître que les facteurs structurels (âge, race, classe sociale et genre) et différents contextes sociaux exercent une influence sur la logigue du risque. Un autre outil de gestion du risque utilisé au Canada, l'échelle de classement par niveau de sécurité, inclut des critères tels que « historique de participation dans des incidents en institution » pour évaluer l'ajustement en institution ${ }^{11}$. Alors que l'inclusion d'un tel critère peut être intuitivement valable, celui-ci demeure très individualiste et ne tient pas compte du contexte.

De la même façon, McHugh (1997) affirme que le concept de risque vise quelque chose à l'intérieur de l'individu, ce qui conduit à une catégorisation sursimplifiée et néglige des questions systémiques plus larges ; il affirme également que les différences de régime et les procédures institutionnelles spécifiques vont influer sur le risque. Par exemple, par ses réactions face à des individus à risque élevé et par sa façon de les

11. Pour une description plus détaillée de la variable comprise dans l'échelle de classement par niveau de sécurité, voir Grant et Luciani, 1998. 
traiter, le personnel peut contribuer à " accroître le risque ». McHugh déclare que pour réduire le risque il est plus important de se concentrer sur l'ambiance et les systèmes d'aide disponibles que de s'en remettre à de «meilleurs outils » et à des programmes sur ordinateur (McHugh, 1997). Cet auteur suggère aussi que les instruments qui servent à prédire le risque, en incorporant les risques et les besoins, n'ajoutent pas grandchose au pouvoir de prédiction, en plus d'être difficiles à compléter et à quantifier.

Dans les connaissances correctionnelles et le calcul du risque, qui se concentrent sur un éventail étroit de corrélations individualisées, on incorpore rarement une perspective sociologique plus large de la stratification sociale, du sexisme, du racisme et de l'application discrétionnaire des règlements. L'opinion des praticiens se forme à partir des normes dominantes sur la culture et le genre ; même lorsque ces praticiens ont reçu une formation de sensibilisation et qu'ils sont conscients des différences de genre et de culture, il leur est difficile d'appliquer ou d'intégrer cette connaissance à leurs évaluations des risques/ besoins. Les évaluations de ces critères sont nettement discrétionnaires et reposent fortement sur des jugements cliniques autant que non cliniques du délinquant. Ces évaluations discrétionnaires surviennent non seulement en présence des variables dynamiques « souples » que nous venons de mentionner, mais aussi en considérant plusieurs des variables prétendues statiques comme « le dossier criminel, l'âge lors du "premier" délit ou "l'emploi légitime" » (Haggerty, 1999). En bref, la rationalité de calcul du risque est discrétionnaire et subjective ; son objectivité, sa cohérence et son efficacité ne sont qu'illusoires. Alors que les facteurs de risque peuvent sembler non moralisateurs et des réalités statistiques neutres révélées grâce à une recherche rigoureusement scientifique, de tels facteurs reflètent également des vues hautement moralisatrices du monde social.

Même si les progrès actuels en matière de technologies d'évaluation du risque et de gestion du risque en rapport avec la gestion des prisonniers semblent plus objectifs et plus efficaces comme méthodes de gestion pénale, les prétentions de ces technologies ne sont souvent pas tout à fait justes. Par exemple, dans la plus grande partie de la recherche fondée sur le risque, les définitions des risques sont statistiques et reflètent les caractéristiques des populations plutôt que des individus. Les facteurs de risque, dérivant de la recherche correctionnelle, reflètent des relations statistiques mais non causales. Quoi qu'il en soit, comme l'a observé O'Malley (1999: 5), on attribue souvent aux individus les 
caractéristiques liées aux catégories de risque (par exemple Sue présente $30 \%$ de chances de récidive) ; les variables identifiées comme facteurs de risque ou servant à prédire le risque selon les statistiques (comme « vit dans un milieu criminogène » ou " chômage » ou « antécédents d'automutilation ») sont interprétées par des praticiens qui évaluent le risque en tant que facteurs causaux. Intentionnel ou non, cet usage abusif et individualisé de l'évaluation du risque vient appuyer les diverses décisions et interventions correctionnelles (incluant plans de programmes, placement institutionnel, transferts, libération conditionnelle, etc). Toutefois, la capacité d'identifier et de quantifier le risque reste essentielle à sa gestion. Une fois les risques identifiés avec succès (i.e. les facteurs menant à la récidive) et établis comme évitables (grâce à des programmes et une planification de la remise en liberté), on est alors en mesure d'assigner la responsabilité de la gestion du risque à des individus ou à des groupes spécifiques.

\section{Pertinence du genre, de la culture et de la position sociale}

Plusieurs des problèmes cités se retrouvent amplifiés lorsqu'on utilise des outils génériques d'évaluation du risque pour gérer des femmes ou des populations carcérales différentes par leur ethnie et leur culture, et souvent plus intensément marginalisées. À quelques exceptions près, les analyses de gestion fondée sur le risque n'ont pas encore exploré en profondeur les aspects relatifs à la classe, au genre et aux dimensions ethno-culturelles de la construction sociale du risque et des pratiques de la gestion du risque. Certains auteurs ont affirmé que le risque est construit en fonction du genre et de la race (Dawson, 1999 ; HannahMoffat, 1999 ; Bhui, 1999 ; Lupton, 1999b).

Des outils comme l'échelle de classement par niveau de sécurité et l'évaluation initiale n'ont pas été conçus pour les femmes, et les évaluateurs n'ajustent pas logiquement leurs critères d'interprétation quand ils les appliquent à elles ${ }^{12}$. Cette remarque revêt un caractère essentiel si l'on admet que la plupart des formes de gestion du risque reposent sur l'iden-

12. Notre recherche pour Condition féminine Canada comprenait une étude détaillée du processus de classification dans les établissements pour femmes condamnées à des peines fédérales au Canada. La façon dont les femmes sont évaluées varie beaucoup selon les régions. Voir Hannah-Moffat et Shaw 2000 pour une discussion détaillée de ces conclusions. 
tification des caractéristiques communes à une population en particulier, et donc que les caractéristiques des populations féminines et des populations ethno-culturelles different de celles de la population pénitentiaire mâle et blanche. Peu d'études du risque tiennent compte des différences au niveau de l'individu ou du groupe sur l'apprentissage du crime ou sur la façon dont le risque et les facteurs de protection peuvent varier d'un groupe à l'autre (Homel, Lincoln, et Herd, 1999 : 183). Ces différences sont particulièrement importantes quand il s'agit de femmes ou de minorités ethniques « pour qui la nature, le sens et l'impact sur la trajectoire de vie des facteurs de protection du risque peuvent différer grandement de ceux de la majorité » (Homel, Lincoln et Herd, 1999 : 183). Toutefois, plutôt que de questionner la pertinence de l'évaluation du risque, certains chercheurs tentent d'établir une série d'éléments de prédiction du risque spécifiques à une culture et qu'on pourrait ajouter aux listes standards ${ }^{13}$ (voir par exemple Homel, Lincoln et Herd, 1999 ; Bonta et al., 1997).

En 1996, on a créé à l'intention des pénitenciers de femmes une série de directives s'appliquant spécifiquement aux femmes pour remplir l'évaluation initiale ; ces directives se basaient sur le manuel de gestion de cas en vigueur et sur « les idées du personnel travaillant dans les institutions pour femmes » (CSC, 1996). Ce document semble incorporer l'information qui met en contexte les circonstances où les femmes tirent un revenu du travail du sexe pour le distinguer, par exemple, d'une source de revenus illégale, et les explications fournies pour l'absence d'expérience de travail ou l'abandon de l'école (i.e. grossesse ou abus physique/sexuel).

Toutefois, peu de personnes présentement responsables de l'évaluation des femmes connaissent l'existence de ce document, et plusieurs ont fait part de leurs difficultés à interpréter et à appliquer les critères de risque «sans égard pour le genre » contenus dans l'évaluation initiale ${ }^{14}$. Des chercheurs canadiens tentent présentement de valider des modèles de classification de sécurité et d'évaluation du risque pour les femmes. Cependant, ce travail ne s'inspire pas de la littérature sur les différences propres au genre du délinquant ou à l'appartenance à une population ethno-culturelle. Il existe peu de discussions ou d'analyses pour savoir comment une

13. Une bonne partie de la recherche au sein du Service correctionnel du Canada tente de répondre à cet objectif. Pour des exemples, voir divers numéros de Forum Recherche sur l'actualité correctionnelle. Cette publication est disponible auprès du Service correctionnel du Canada dans les deux langues officielles.

14. Voir Hannah-Moffat et Shaw, 2000. 
plus forte réponse des institutions ou des gestes posés par le personnel affectent le comportement des prisonniers ou les variables structurelles $\mathrm{du}$ crime et de la récidive chez les femmes (Loucks et Zamble, 1999; Motiuk et Blanchette, 1998 ; Blanchette, 1997a, 1997b, 1997c ; Blanchette et Motiuk,1995, 1997 ; Motiuk, 1997 ; Couslon, Ilacqua, Nutbrown, Giulekas et Cudjoe, 1996c). Bien que nous accueillons favorablement cette volonté de reconnaitre la différence, nous soutenons que la pratique générale de " validation » des barèmes actuels d'évaluation du risque et du besoin, basés sur la recherche et les théories applicables à la criminalité masculine, est théoriquement et empiriquement problématique lorsque appliquée à la population féminine.

On a déjà examiné en relation avec la violence les problèmes découlant de l'utilisation pour les femmes de catégories ne tenant pas compte du genre (Dougherty, 1993 ; Campbell, 1993 ; Shaw et Dubois, 1995 ; Comack, 1996, 1998 ; Jackson, 1999). Au compte de ces problèmes, mentionnons le manque de cohérence des définitions de la violence dans la littérature, l'éventail des comportements considérés violents, les restrictions des catégories officielles qui « considèrent en bloc » divers comportements, et l'importance du contexte culturel qui façonne notre compréhension et notre définition de la violence. La littérature courante sur la santé mentale ou la violence, par exemple, s'appuie sur des échantillons très sélectifs et de très petits nombres de femmes. Les comparaisons entre les hommes et les femmes sont aussi embrouillées par le traitement différentiel qui est fait entre hommes et femmes par la police et les tribunaux. Plus important encore, la plus grande partie de la littérature sur le traitement, tout comme sur la classification, s'appuie sur des études portant sur les hommes et généralisées aux femmes, quand celles-ci ne sont pas complètement ignorées. Troisièmement, différentes disciplines tendent à expliquer et à interpréter le comportement de diverses manières : les explications d'ordre sociologique tiennent compte des influences plus larges sur le comportement, alors que les explications psychologiques mettent l'accent sur le développement individuel ou la pathologie. Les systèmes correctionnels qui mettent l'accent sur la pathologie individuelle ont tendance à accorder moins d'importance au contexte.

La recherche selon le genre suggère également le besoin de dépasser le simple ajout d'une autre variable à un barème du risque ou à une évaluation des besoins. L'adaptation de mesures actuarielles dérivant des hommes n'élimine pas nécessairement la neutralité par rapport au genre ou son ignorance. Le simple fait d'ajouter des barèmes d'évaluation du 
risque et du besoin pour inclure des critères tenant compte du genre ne reflète pas adéquatement l'étendue des différences entre les hommes et les femmes, ou entre les femmes elles-mêmes. Cette conception tient pour acquis que tous les critères d'évaluation, mis à part quelques-uns, sont les «mêmes » pour les hommes et les femmes, sans contester les éléments établis selon le sexe ou la race des données empiriques sur lesquelles l'instrument est construit au point de départ.

Présentement, pour établir des programmes d'intervention efficaces qui répondent aux besoins des femmes délinquantes et qui parviennent à diminuer leur risque de récidive, on affirme qu'il faut considérer le profil démographique et l'historique de la population féminine, ainsi que l'impact des divers facteurs de vie sur leur criminalité (Bloom et Covington, 1998 ; Abbott et Kerr, 1995) ${ }^{15}$. Les caractéristiques de la population féminine sont relativement bien établies. Certaines de ces caractéristiques sont communes aux deux sexes, d'autres non. Par exemple, les femmes délinquantes sont souvent victimes d'abus physiques, sexuels ou autres infligés par des intimes ou des connaissances. Elles sont souvent mères et les premières responsables des soins, leur bagage académique est limité, elles sont plus souvent que les hommes sans emploi au moment de leur délit $^{16}$, et beaucoup vivent dans la dépendance financière. Nombreuses sont celles qui ont une dépendance aux drogues ou à l'alcool, sans parler des problèmes de santé physique ou mentale.

On a aussi une assez bonne compréhension des différences quantitatives au sujet du nombre et des types de délits commis par les femmes ${ }^{17}$. Ce que l'on comprend moins bien, ce sont les différences contextuelles et les différences qualitatives selon le genre qui existent entre délits et délinquants. Alors qu'il existe peu d'études détaillées sur la délinquance féminine (et encore moins à l'échelle canadienne), l'évidence actuelle suggère

15. Une grande proportion de féministes spécialistes de la question prétendent que l'apprentissage est fonction du genre et de la race à cause d'une socialisation différentielle sur la base du genre et fort probablement de la race, de l'ethnie et de la culture (Morris, 1987). Des recherches récentes suggèrent des différences dans la façon dont les hommes et les femmes apprennent, abordent le monde social et interagissent avec autrui (Bloom et Covington, 1998 ; voir aussi Zaplin, 1998).

16. Une proportion significativement plus élevée de femmes que d'hommes étaient sans emploi au moment de leur arrivée en prison. Les données disponibles révèlent que $64 \%$ des détenues dans les établissements provinciaux / territoriaux et $80 \%$ des détenues dans les pénitenciers fédéraux étaient sans emploi au moment de leur admission comparativement à respectivement $43 \%$ et $54 \%$ chez les hommes (Finn, Trevethan, Carrière, Kowalski, 1999 : 5 ).

17. Pour des exemples détaillés sur ces différences voir entre autres Boritch, 1997 ; Johnson, 1986 ; in Finn, Trevethan, Carrière, Kowalski, 1999 : 2. 
que la nature du délit chez la femme est qualitativement différente de celle de l'homme, même si les accusations sont les mêmes. Les activités criminelles dans lesquelles sont impliquées les femmes, le chemin qui les mène au crime, leurs modes d'ajustement en institution, ainsi que les risques d'évasion sont différents. En bref, la recherche récente sur la délinquance féminine semble indiquer que le crime est une activité fortement spécifique et que la motivation au crime, le contexte du délit, l'accès aux occasions de crime, de même que les réactions à la prison, sont façonnés par les différences de styles de vie que mènent les hommes et les femmes (cf. Steffensmeier et Allan, 1999). Puisque très peu d'études au Canada se sont penchées sur ces différences, notre connaissance des causes de la délinquance des femmes est limitée. Il s'ensuit que, vu l'absence d'études qualitatives autant que quantitatives sur les modèles de la délinquance féminine, nos outils d'évaluation et notre capacité à élaborer des programmes sont aussi fondamentalement limités. En termes de première offense et de récidive, et vu les vastes fluctuations dans les populations de femmes délinquantes de même que dans les systèmes de justice, la recherche menée en Amérique du Nord n'a pas établi un modèle de causalité qui produise des résultats qui se tiennent.

Ce problème s'amplifie quand on considère les différences culturelles ou ethniques. Si l'on connaît peu de choses au sujet des femmes délinquantes, on en sait encore moins sur les différences qualitatives entre délinquants blancs et non blancs. Un vaste segment de la recherche critique le concept d'une «femme universelle » et affirme que l'oppression et les différences culturelles contribuent à accentuer les différences qualitatives et quantitatives entre les femmes délinquantes ${ }^{18}$. Jackson (1999), par exemple, soutient que le croisement de la race, du genre et de la classe forme un contexte spécial chez les femmes autochtones, enraciné dans un héritage colonial de politiques d'assimilation. De plus, alors qu'on possède un peu d'information sur la dynamique et les différences contextuelles entre les expériences et les histoires des femmes autochtones et les non autochtones ${ }^{19}$, on en sait considérablement moins sur les femmes

18. Pour une description détaillée de certaines des études canadiennes qui mettent en doute le concept de femme universelle, voir Bouchard, Boyd et Sheehy, 1999.

19. La connaissance de ces différences est essentielle, vu que les femmes autochtones constituent environ $30 \%$ de toutes les femmes admises dans des institutions provinciales ou territoriales (Lipinski, 1991), et environ 19 \% des détenues dans les pénitenciers fédéraux (Arbour, 1996). Cette situation est stupéfiante étant donné que les Autochtones ne composent qu'environ $3 \%$ de toute la population. Les besoins culturels et spirituels des 
noires ou provenant d'autres minorités dans le système de justice criminelle canadien, et les modèles contextuels de leurs délits. Toutefois, des rapports gouvernementaux récents comme le Report of the Ontario Commission on Systemic Racism et le Report of the Task Force On Aboriginal Peoples révèlent que l'on néglige souvent les besoins de ces groupes, et qu'ils subissent diverses formes de racisme direct et systémique et de discrimination.

Le rapport Arbour (1996) réitère que les femmes et les minorités culturelles ont des besoins et des caractéristiques qui different de ceux des hommes ; c'est pourquoi l'approche se doit d'être différente. Au sujet des femmes autochtones, ce rapport souligne six problèmes spécifiques (p. 218-219) : leur sur-représentation dans la population carcérale ; le caractère distinctif de leur culture, leur langue et leurs habitudes sociales par rapport aux autres femmes dans les pénitenciers; la différence marquante de leur histoire personnelle et sociale ; la différence significative quant à leurs délits ; le fait que l'éloignement géographique soit un fardeau particulier ; et leur approche holistique de la guérison et de la réintégration qui va à l'encontre de la philosophie et de la culture traditionnelles des prisons. Le rapport Arbour soutient que ces problèmes ont été enfouis dans un environnement carcéral qui se trouve à l'opposé de bien des cultures autochtones (p. 220). L'effet cumulatif de leurs dossiers criminels plus considérables, marqués d'un plus grand nombre de délits de violence et d'incarcérations que pour les femmes non autochtones, entraîne généralement une classification de sécurité et une évaluation du risque plus élevées, «alourdies par les tensions et les malentendus qui existent entre les cultures autochtones d'une part, et la justice criminelle et les milieux pénaux d'autre part » (p. 221). Le rapport conclut qu'il existe plus de différences que de similitudes entre les délinquants hommes et femmes. «Leurs crimes sont différents, leurs facteurs criminogènes sont différents, et leurs besoins correctionnels en matière de programmes et de services sont différents. Plus important encore, le risque que les femmes posent pour la société, en tant que groupe, est minime, et considérablement différent du risque de sécurité posé par les hommes » (Arbour, $1996: 228$ ).

\footnotetext{
femmes autochtones different de ceux des femmes blanches ; on retrouve aussi plus de foyers monoparentaux, de problèmes familiaux et de placements en foyers d'accueil chez les premières (Caswey, Bear, Bertolin, Cooper, Frenklin, Galet et Gallagher, 1991 ; Correctional Law Review, 1988 ; Commission royale sur les peuples autochtones, 1996) ; et une plus grande fréquence de carences économiques et sociales associées à des expériences de racisme (Commission royale sur les peuples autochtones, 1996).
} 
D'autres auteurs ont aussi critiqué l'accent mis sur le risque et l'utilisation de mesures actuarielles. Dans les années 1980, on a soulevé des questions relativement à leur impact sur les minorités, en particulier sur les mâles américains noirs, mais les discussions relatives au genre des délinquants ont été pratiquement nulles. Certains ont affirmé que les applications rigides et mécaniques fournies par les outils d'évaluation pouvaient mener à des classifications non équitables (Petersilia et Turner, 1987). D'autres ont mis en garde contre l'institutionnalisation des désavantages infligés aux minorités (Gottfredson, 1987). Dans le contexte canadien, mentionnons parmi ces désavantages : le racisme, les expériences dans les internats, le taux élevé de chômage, l'analphabétisme, l'alcoolisme, les exigences culturelles conflictuelles, et les différences des conditions régionales, des expériences de vie et des structures sociales dans les communautés autochtones.

D'autres documents suggèrent que même des concepts aussi fondamentaux que la nature et le sens du crime peuvent varier dans certaines communautés autochtones. Monture (2000 : 56) considère les échelles de risque comme des instruments individualisés qui n'arrivent pas à tenir compte de la portée de l'oppression coloniale dans la vie des Autochtones, hommes et femmes, comme sur leurs communautés et nations. Elle affirme que la nature individualisée de la loi masque les facteurs systémiques et structurels ; elle soutient également que ce problème est aussi présent dans les tribunaux que dans les autres processus de prise de décision comme la classification de sécurité, l'évaluation du risque, le placement dans les pénitenciers et la libération conditionnelle (Monture, 2000 : 57). Malgré la volonté croissante de mettre au point des systèmes de classification adaptés aux femmes, nombre de recherches et de discussions sur la classification et la prédiction du risque, dans les années 1990, n'ont pas pris en compte le genre et la diversité. Parfois on ne fait aucune distinction entre femmes et hommes ; d'autres fois, on ne mentionne même pas le genre masculin des sujets (voir Dhaliwal et al., 1994 ; Clement, 1996 ; Holt, 1996 ; Aubrey et Hough, 1997 ; Quinsey et al., 1998).

Les différences quantitatives et qualitatives sont primordiales et elles ont un impact sur les interprétations institutionnelles $\mathrm{du}$ « risque » et, en bout de ligne, sur les pratiques de gestion du risque. Bien des problèmes d'ordre théorique, statistique et méthodologique surgissent quand on ignore le genre du délinquant. Le modèle de risque/réhabilitation de gestion pénitentiaire contribue directement aussi bien qu'indirectement aux pratiques discriminatoires dans l'identification et la gestion des 
besoins, et sur toutes les décisions fondées sur de telles évaluations. Ancrés dans des évaluations neutres du risque, on trouve des critères qui ne reflètent pas la réalité sociale des populations carcérales spécifiques, et qui représentent une marginalisation ainsi que des désavantages sociaux décontextualisés. Notre souci principal concerne le fait que, à l'heure actuelle, la compréhension et les constructions sociales du risque et de sa gestion ne prennent pas conscience des inégalités structurelles plus larges et des différences systémiques au sein de ces groupes. Il faut établir une analyse plus poussée de l'interprétation du risque en fonction des contextes socio-culturels et voir comment ces contextes façonnent le discours sur le risque.

\section{Conclusions}

Nous espérons que cet article stimulera un débat sérieux sur les pratiques de gestion fondée sur le risque telles qu'appliquées dans le milieu correctionnel au Canada, en particulier face aux populations carcérales féminines ou non blanches. Nous espérons également contribuer à l'amélioration de la spécificité du genre et de l'ethno-culture dans la recherche, les politiques et les opérations correctionnelles dans leur rapport avec la classification de sécurité et l'évaluation du risque/des besoins. Il est important d'établir des liens plus concrets entre la théorie, la recherche, les politiques et les pratiques correctionnelles, et la relation qui existe entre ces sphères pour établir comment le risque est opérationnalisé et géré.

Nous soutenons qu'un modèle mixte de gestion (réhabilitation/ risque) prévaut dans les milieux correctionnels canadiens. Ce modèle de gestion repose fortement sur l'hybridation des concepts de risque et de besoin, et joue un rôle essentiel dans la réaffirmation d'un système de sentence basé sur la réhabilitation.

Les connaissances sur le risque fournissent de l'information pour les pratiques correctionnelles. Alors que les facteurs de risque constituent des artefacts statistiques qui reflètent certaines caractéristiques de population, il arrive souvent que l'on attribue ces facteurs à des individus et qu'on les utilise pour légitimer un vaste éventail d'interventions, y compris la responsabilisation des délinquants. Cette attribution du risque à des individus découle du développement d'outils d'évaluation, ainsi que de l'administration et de l'interprétation desdits outils. Nos observations sur ce processus révèlent des tendances inquiétantes qui 
demandent que l'on procède à d'autres examens critiques des critères à la base des technologies d'évaluation du risque et des méthodes proposées pour la réduction du risque. Les chercheurs et les praticiens qui se font les défenseurs des technologies du risque définissent souvent le risque en se fondant sur une moralité de classe moyenne de race blanche. Subtilement, on institutionnalise et on reproduit les désavantages sociostructurels autant que les stéréotypes basés sur le genre et les dimensions ethno-culturelles. À première vue, le risque et l'initiative de sa gestion apparaissent non moralisateurs, efficaces, objectifs et non discriminatoires ; mais tout autre est la réalité.

\section{Références}

Aвbott, B. et KerR, D. 1995. Substance Abuse Program for Federally Sentenced Women, Ottawa : Correctional Service Of Canada.

Aboriginal Justice InQuiry OF Manitoba. 1991. Report of the Aboriginal Justice Inquiry of Manitoba, Winnipeg : Queen's Printer.

ANDrews, D., BonTA, J. et HogE, R.D. 1990. «Classification for Effective Rehabilitation. » Criminal Justice and Behaviour 17 : 19-52.

Andrews, D., Zinger, I., Hoge, R.D., Bonta, J., Gendreau, P. et Cullen, F.T. 1990. «Does Correctional Treatment Work? A Clinically Relevant and Psychologically Informed Meta-Analysis » Criminology 28 (3) : 369-404.

ARbOUR, L. 1996. Report of the Commission of Inquiry Into Certain Events at the Prison for Women in Kingston, Ottawa : Public Works and Government Services.

Aubrey, R. et Hough, M. 1997. Assessing Offender's Needs: Assessment Scales for the Probation Service, Home Office Research Study 166, London : Home Office.

BHuI, H. S. 1999. « Racism and Risk Assessment: Linking Theory to Practice with Black Mentally Disordered Offenders » Probation Journal 46 (3) : 171 181.

Blanchette, K. 1997a. «Classifying Female Offenders for Correctional Interventions » Forum on Corrections Research 9 (1), Ottawa : Correctional Service of Canada.

Blanchette, K. 1997b. « Comparing Violent and Non-Violent Offenders on Risk and Need » Forum on Corrections Research 9 (2), Ottawa : Correctional Service of Canada.

BlANCHETTE, K. 1997c. Risk and Need Among Federally-Sentenced Female Offenders: A Comparison of Minimum-Medium-and Maximum-Security Inmates, Research Report No. R-58, Ottawa : Correctional Service of Canada.

Blanchette, K. et Motiuk, L. 1995. Female Offenders Risk Assessment: The Case Management Strategies Approach, Paper Presented at the Canadian Psychological Association Annual Convention. Charlottetown. 
Blanchette, K. et Motiuk, L. 1997. « Maximum Security Female and Male Federal Offenders. A Comparison » Forum on Corrections Research 9 (3), Ottawa : Correctional Service of Canada.

Bloom, B. et Covington, S. 1998. Gender-Specific Programming for Female Offenders: What Is It and Why It Is Important?, Paper Presented at The American Society of Criminology Meetings, Washington, DC, November.

Bonta, J., Pang, B. et Wallace-Capretta, S. 1995. "Predictors of Recidivism Among Incarcerated Females Offenders » The Prison Journal 75 (3) : 227-293.

Bonta, J., Laprairie, C. et Wallace-Capretta, S. 1997. «Risk Predictors and Re-Offending: Aboriginals and Non-Aboriginal Offenders " Canadian Journal of Criminology 39 (2) : 127-144.

BORITCH, H. 1997. Fallen Women: Female Crime and Criminal Justice in Canada, Toronto : ITP Nelson.

Bouchard, J., Boyd, S. et SheeHy, L. 1999. « Canadian Feminist Research on Law: An Annotated Bibliography » Canadian Journal of Women and The Law $11(1-2)$.

Campbell, A. 1993. Men, Women and Aggression, Basic Books.

CASTEL, R. 1991 « From Dangerousness To Risk », in The Foucault Effect Studies in Governmentality, sous la direction de G. Burchell, C. Gordon et P. Miller. Chicago : University of Chicago Press.

Caswey, R., Bear, W. L., Bertolin, C., Cooper, C., Frenklin, J., Galet A. et Gallagher, M. 1991. Justice on Trial: Report of The Task Force on The Criminal Justice System and Its Impact on Indian, Metis People of Alberta, Alberta.

Canadian Criminal Justice Association. 1998. Prison Overcrowding and the Reintegration of Offenders, Ottawa, March.

Clark, D.A., Fisher, M. J. et MCDOugall, C. 1993. « A New Methodology for Assessing Level of Risk in Incarcerated Offenders » British Journal of Criminology 33 (3) : 436-448.

Clements, C.B. 1996. «Offender Classification. Two Decades of Progress » Criminal Justice And Behaviour 23 (1) : 121-143.

COMACK, E. 1996. Women in Trouble, Halifax : Fernwood Publishing.

COMACK, E. 1998. Gendered Violence: Men's and Women's Violence in the Criminal Justice System, Unpublished Paper presented at Workshop on Women and the Criminal Justice System, Peterborough : Trent University, May 1999.

Correctional Law Review. 1988. Correctional Issues Affecting Native Peoples. Ottawa : Ministry of the Solicitor General.

Correctional Service Of CANADA -CSC 1996. FSW Facilities Offender Intake Assessment Content Guidelines, September, Ottawa : Correctional Service of Canada.

Coulson, G., Ilaceua, G., Nutbrown, V., Giulekas, D. et Cudjoe, F. 1996. "Predictive Utility of the LSI for Incarcerated Female Offenders » Criminal Justice Behaviour 23(3) : 427-439. 
CulPitT, I. 1999. Social Policy and Risk, London : Sage Publications.

DallaO, M. 1997. «Keeping Classification Current » Corrections Today July : 86-88.

Dawson, D. 1999. Risk of Violence Assessment: Aboriginal Offenders and Assumption of Homogeneity, Paper Presented at the Best Practice Interventions in Corrections for Indigenous People Conference, Adelaide, Australia. Australian Institute of Criminology and Department of Correctional Service For S.A.

DeAn, M. 1999. Governmentality: Power and Rule in Modern Society, London : Sage Publications.

Defert, D. 1991. "Popular Life and Insurance Technology », Pp. 211-234 in The Foucault Effect: Studies in Governmentality, sous la direction de G. Burchell, C. Gordon et P. Miller. Chicago : University Of Chicago Press.

Dhaliwal, G.K., PORPorino, F. et Ross, R.R. 1994. « Assessment of Criminogenic Factors, Program Assignment, and Recidivism » Criminal Justice and Behaviour 21 (4) : 454-467.

Ditchfield, J. 1997. « Actuarial Prediction and Risk Assessment » Prison Service Journal $113: 8-13$.

DOUgherTY, J. 1993. «Women's Violence Against Their Children: A Feminist Perspective » Women and Criminal Justice 4 (2) : 91-114.

ERICSON, R. et HagGerty, K. 1997. Policing the Risk Society, Toronto : University of Toronto Press.

EWALD, F. 1991. «Insurance and Risk », in The Foucault Effect Studies in Governmentality, sous la direction de G. Burchell, C. Gordon et P. Miller. Chicago : University of Chicago Press.

Feeley, M. et Simon, J. 1992. "The New Penology: Notes on the Emerging Strategy for Corrections and Its Implications » Criminology 30 (4) : 49-74.

FeEley, M. et SimON, J. 1994. « Actuarial Justice: The Emerging New Criminal Law », in The Futures of Criminology, sous la direction de D. Nelken. London : Sage.

Federally Sentenced Women's Program - FSWP. 1994. Literature Review, Ottawa : Correctional Services.

Finn, A., Trevethan, S., Carrière, G. et Kowalski, M. 1999. «Female Inmates, Aboriginal Inmates and Inmates Serving Life Sentences: A One Day Snapshot » Juristat 19 (5) : 1-5, Ottawa : Statistics Canada.

GARLAND, D. 1996. "The Limits of the Sovereign State: Strategies of Crime Control in Contemporary Society » British Journal Of Criminology 36 (4) : 445-471.

GaRLAND, D. 1997. "Governmentality and the Problem of Crime: Foucault, Criminology, Sociology » Theoretical Criminology 1 (2) : 173-214.

Gendreau, P., GogGon, C. et Gray, G. 1998. « Case Need Domain: "Employment" » Forum on Corrections Research 10 (3), Ottawa : Correctional Service of Canada. 
GotTfredson, D. M. et TONRY, M. 1987. «Prediction and Classification » Crime and Justice 9.

Grant, B. et LuCiani, F. 1998. Security Classification Using the Custody Rating Scale, Ottawa : Research Branch - Correctional Service of Canada.

Haggerty, K. 1999. Correctional Risk Classifications: Pragmatic Cautions and Theoretical Reflections, Paper presented at workshop on Risk Gender and Diversity, Toronto : Status of Women Canada.

Hannah-Moffat, K. 1999. «Moral Agent or Actuarial Subject: Risk and Canadian Women's Imprisonment » Theoretical Criminology 3 (1) :71-94.

Hannah-Moffat, K. 2000. «Re-Forming the Prison- Rethinking Our Ideals ", in An Ideal Prison?: Critical Essays on Women's Imprisonment in Canada, sous la direction de K. Hannah-Moffat et M. Shaw. Halifax : Fernwood.

Hannah-Moffat, K. et Shaw, M. 2000. Gender, Diversity, Risk Assessment and Classification with Federally Sentenced Women, Ottawa: Status of Women Canada.

Holt, N. 1996. Inmate Classification: A Validation Study of the California System, California Department of Corrections.

Homel, R., Lincoln, R. et Herd, B. 1999. « Risk and Resilience: Crime and Violence Prevention in Aboriginal Communities » Australian and New Zealand Journal of Criminology 32 (2) : 182-196.

JACKSON, M. 1999. «Canadian Aboriginal Women and their 'Criminality': The Cycle of Violence in the Context of Difference » Australian and New Zealand Journal of Criminology 32 (2) : 197-208.

LIPINSKI, S. 1991. « Adult Female Offenders In Provincial / Territorial Correctional Systems 1989-90 » Juristat 11 (6), Canadian Centre for Justice Statistics, Statistics Canada.

LOUCKS, A. et ZAMBLE, E. 1999. « Predictors of Recidivism: In Serious Female Offenders - Canada Searches for Predictors Common to Both Men and Women » Corrections Today February : 26-32.

Luhmann, N. 1993. Risk: A Sociological Theory, New York : Aldine De Gruyter.

LUPTON, D. 1999a. Risk, London : Routledge.

LuPTON, D. 1999b. Risk and Socio-cultural Theory: New Directions and Perspectives, Cambridge : Cambridge University Press.

MCHugh, M. 1997. «Risk Assessment and Management of Suicides in Prison » Prison Service Journal $113: 4-8$.

MotiuK, L. 1993. «Where Are We in Our Ability to Assess Risk? » Forum on Corrections Research 5 (2), Ottawa : Correctional Service of Canada.

MOTIUK, L. 1997. « Classification for Correctional Programming: the Offender Intake Assessment (OIA) Process » Forum on Corrections Research 9 (1), Ottawa : Correctional Service of Canada.

Motiuk, L. et Blanchette, K. 1998. What Works in Assessing Female Offender Risk and Need, Paper Presented at ICCA $6^{\text {th }}$ Annual Research Conference, Arlington Virginia. 
Monture, P. 2000. "Aboriginal Women and Correctional Practice ", in $A n$ Ideal Prison?: Critical Essays on Women's Imprisonment in Canada, sous la direction de K. Hannah-Moffat et M. Shaw. Halifax : Fernwood.

O'Malley, P. 1992. «Risk, Power and Crime Prevention » Economy and Society $21(3): 252-275$.

O'Malley, P. 1998. Crime and the Risk Society, Sydney : Ashgate Dartmouth.

O'MALLEY, P. 1999. The Risk Society Implications for Justice and Beyond, Report Commissioned for the Department of Justice, Victoria, Australia.

Parton, N. 1996. Social Theory Social Change and Social Work, New York: Routledge.

PRATT, J. 1993. «Dangerousness, Risk and Technologies of Power » Australian and New Zealand Journal of Criminology $28: 3-31$.

PRATT, J. 1997. Governing the Dangerous, Sydney: The Federation Press.

Petersilia, J. et Turner, S. 1987. "Prediction and Racial Minorities », in Prediction and Classification, sous la direction de D.M. Gottfredson et M. Tonry. Crime and Justice 9, Chicago : University Of Chicago Press.

PRICE, R. 1997. "On The Risks of Risk Prediction " Journal of Forensic Psychiatry 8 (1) : 1-4.

QUinSEY, V., HARRIS, G.T., RICE, M.E. et CORMIER, C.A. 1998. Violent Offenders: Appraising and Managing Risk, Washington DC: American Psychological Association.

Royal Commission Of AbORIginal PeOples. 1996. Bridging the Cultural Divide Report on Aboriginal Peoples In The Criminal Justice System, Ottawa : Ministry of Supply and Service.

Shaw, M. et DubOIS, S. 1995. Understanding Violence by Women, Ottawa : Correctional Service of Canada.

SimON, J. 1987. "The Emergence of a Risk Society: Insurance, Law and the State » Socialist Review 95 (1) : 93-108.

SimON, J. 1988. "The Ideological Effects of Actuarial Practices » Law and Society Review 22 (4) : 771-800.

Simon, J. et Feeley, M. 1995. « True Crime: The New Penology And Public Discourses On Crime », Pp. 147-180 in Punishment And Social Control, sous la direction de T. Blomberg et S. Cohen. New York : Aldine De Gruyter.

Steffensmeier, D. et Allan, E. 1998. " Nature of Female Offending: Patterns and Explanation », in Female Offenders Critical Perspectives and Effective Interventions, sous la direction de R. Zaplin. Maryland : Aspen Publishers Inc.

ZAPLIN, R.T. 1998. Female Offenders: Critical Perspectives and Effective Interventions, Maryland : Aspen Publishers. 